\title{
Stellenwert der Januskinasen
}

„Rheuma“ ist ein Sammelbegriff für über 400 verschiede entzündliche Immunerkrankungen. Beinahe jeder vierte Österreicher ist Rheuma-Patient. Mit Januskinase (JAK)-Inhibitoren steht seit rund drei Jahren eine Therapieoption der rheumatoiden Arthritis (RA) zur Verfügung, die aktuell besonders bezüglich Remission hervorragende Ergebnisse erzielt. Fragen rund um neue Therapie-Optionen diskutierten Rheumatologe Priv. Doz. Dr. Johannes Grisar und Dr. Isabella Presch, Medical Director bei AbbVie in Österreich, im Rahmen einer Pressekonferenz.

Zwischen 70.000 und 80.000 Österreicher, drei Viertel davon Frauen, leiden an rheumatoider Arthritis, der häufigsten chronisch-entzündlichen Systemerkrankung. Kriterien wie mehr als zwei geschwollene Gelenke und eine Morgensteifigkeit von über einer Stunde gelten als typische Verdachtsmerkmale. Begleitet werden diese Symptome häufig von Schwäche, Müdigkeit und einem anhaltenden Krankheitsgefühl.

Bei der rheumatoiden Arthritis kommt es aufgrund einer Fehlsteuerung des Immunsystems zu einer Entzündung der inneren Schichten der Gelenkskapsel, die eine Zerstörung der Gelenksknorpel bzw. des gesamten Gelenkes zur Folge haben kann. Diese Gelenksentzündungen können wiederkehrend oder ständig auftreten.

Sehr häufig steht bei der Behandlung der rheumatoiden Arthritis die Milderung der Symptome, des Schmerzes und der Schwellungen von Gelenken im Vordergrund. Betroffene sehen demnach ihre Erkrankung als "gut behandelt" oder "unter Kontrolle", wenn sie schmerzfrei sind. Bei einem zielorientierten Vorgehen geht es darum, Patienten zu helfen, eine geringe Krankheitsaktivität zu erreichen.

\section{Mittler zwischen „,außen“ und ,innen“}

Mit den Januskinase-Inhibitoren steht seit geraumer Zeit eine moderne Option in der Therapie der rheumatoiden Arthritis zur Verfügung. JAK-Inhibitoren werden als targeted synthetic (ts) DMARDs bezeichnet und sind in mehreren Parametern Biologika übergeordnet.

Januskinasen sind zytoplasmatische Tyrosinkinasen, die an Zytokin-Rezeptoren binden. Zytokin-Rezeptoren besitzen keine eigene Enzymaktivität und benötigen daher die Kinasen JAK1, JAK2, JAK3 oder TYK2 (Tyrosinkinase 2), um intrazelluläre Signalkaskaden zu aktivieren. Nach Aktivierung über so genannte STAT(Signal Transducer and Activator of Transcription)Proteine vermitteln sie Effekte ins Zellinnere. Bindet im Extrazellulärraum ein Zytokin an seinen Rezeptor, wird dieses Signal intrazellulär wahrgenommen und weitergegeben.

\section{Januskinasen sind Team-Player}

Januskinasen entfalten ihre Funktion nur als Tandem. Je zwei Mitglieder der JAK-Familie, die aus den vier Proteinen JAK1, JAK2, JAK3 und TYK2 besteht, bilden ein Paar, das spezifisch für eine Gruppe von Zytokinrezeptoren ist. Ohne JAK-Aktivität kann das Signal, das im Extrazellulärraum auf Zytokinrezeptoren trifft, nicht ins Zellinnere und in den Zellkern gelangen. Damit wird die Kaskade unterbrochen, was bei verschiedenen Krankheiten wie etwa der rheumatoiden Arthritis therapeutisch erwünscht ist.

Zu den Vorteilen der JanuskinaseInhibitoren zählt, dass sie in ihrer Handhabung unkompliziert sind. Sie müssen weder gekühlt noch gespritzt werden und können im Unterschied zu vielen anderen Therapien auch als Monotherapie angewendet werden. Neben der entzündungshemmenden Funktion verhindern Januskinase-Inhibitoren viele Begleitsymptome wie Müdigkeit oder Schmerzen und weisen deutlich höhere Ansprechraten auf als andere Therapieformen. Das Ziel der RheumaRemission kann so schneller und häufiger erreicht werden.

\section{Remission ist möglich}

Rheuma ist nicht heilbar, es ist aber möglich, eine Remission zu erreichen, bei der die Symptome kontrollierbarer sind und sich nicht auf den Alltag der Patienten auswirken. Medizinisch bedeutet das eine effektive Krankheitskontrolle und Symptomlinderung. "Eine Remission ist nur möglich, weil moderne Medikamente gezielt ins Krankheitsgeschehen eingreifen und die entzündungsverursachenden Prozesse im Körper hemmen. Besonders mit den Januskinase-Hemmern erreichen Patienten in nur wenigen Wochen einen langanhaltenden Krankheitsstillstand und die Möglichkeit, ein normaleres Leben führen zu können," so Priv. Doz. Dr. Grisar, Leiter der Rheumaambulanz im Sanatorium Hera.

Hinweis des Verlags. Der Verlag bleibt in Hinblick auf geografische Zuordnungen und Gebietsbezeichnungen in veröffentlichten Karten und Institutsadressen neutral.

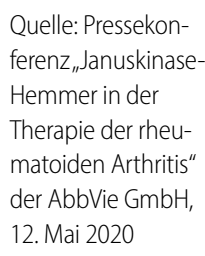

$\begin{array}{ll}\text { rheuma plus 2020 } 19: 91 & \text { Hemmer in der } \\ \text { https://doi.org/10.1007/s12688-020- } & \text { Therapie der rheu- } \\ \text { 00349-z } & \text { matoiden Arthritis" } \\ \begin{array}{l}\text { ○ Springer-Verlag GmbH Austria, ein Teil } \\ \text { von Springer Nature 2020 }\end{array} & \text { der AbbVie GmbH, } \\ \text { 12. Mai } 2020\end{array}$
von Springer Nature 2020 